\title{
Postural changes in perfusion pressure and retinal arteriolar calibre
}

\author{
S HAGUE AND D W HILL \\ From the Research Department of Ophthalmology, Royal College of Surgeons of England, and Moorfields Eye \\ Hospital, London
}

SUMMARY Ophthalmic artery perfusion pressures and retinal arteriolar calibres were studied in 10 human subjects when sitting, standing, and lying. Differing responses in perfusion pressure were found; autoregulatory responses were found in 13 of the 18 significant calibre changes on moving from sitting to standing and 13 of 22 significant calibre changes on moving from standing to lying. The remaining significant calibre changes were non-autoregulatory, the arterioles dilating with perfusion pressure increase and constricting with decrease.

Autoregulation, the intrinsic tendency to maintain constant blood flow despite changes in perfusion pressure, occurs in many tissues, protecting their metabolic requirements.

Changes in posture are associated with alterations in perfusion pressure. On standing, arterial blood pressure at the head drops by $20-30 \mathrm{mmHg}$ and the jugular venous pressure decreases by $5-8 \mathrm{mmHg}$. Cerebral vascular resistance also falls because of the decrease in intracranial pressure. The resulting decrease in cerebral blood flow leads to an increase in local $\mathrm{PCO}_{2}$ and a decrease in $\mathrm{Po}_{2}$ and $\mathrm{pH}$. These metabolic changes lead to vasodilatation and an associated restoration of adequate blood flow. The constancy of cerebral blood flow over a wide variety of conditions is a striking feature of its physiology. ${ }^{1}$

It would seem reasonable to speculate that the retina, sharing a common embryological origin and a high metabolic demand, should show a similar capacity to autoregulate. Support for this hypothesis has been given by a small number of studies of the human retinal circulation. Russell ${ }^{2}$ reported an increase in retinal arterial calibre in response to an acute increase in intraocular pressure induced by applying pressure to the globe. This occurred both in patients with a normal vascular innervation and in those with Horner's syndrome. Dobree ${ }^{3}$ found that, when intraocular pressure was decreased either by drugs or by operation in patients with glaucoma,

Correspondence to Miss S Hague, FRCS, Research Department of Ophthalmology, Royal College of Surgeons, Lincoln's Inn Fields, London WC2A 3PN. retinal arterial and venous constriction occurred. Riva and Loebl, ${ }^{4}$ using the blue light entoptic phenomenon to measure human macular blood flow, recorded autoregulation in response to a change in perfusion pressure. The average time lag of the response was 46 seconds and the duration of the response was about 48 seconds.

In this study posture was chosen as a physiological stimulus to an alteration of perfusion pressure. The circulatory problems of the eye are unique. The venous pressure elsewhere in the head and neck becomes negative with respect to atmospheric pressure on standing. Gravity would be assumed likewise to decrease venous pressure in the eye, but this must always be maintained above the intraocular pressure. ${ }^{5}$

It is of additional interest to study the retinal vasculature in relation to posture, since many retinal venous occlusions present in the morning after lying down overnight. In addition such patients often notice a diurnal variation in their vision, seeing less well in the morning. Pathologically high increases in intraocular pressure on adopting a supine posture have been associated with retinal vein obstructions ${ }^{6}$ and suggested as a high risk factor for the development of chronic simple glaucoma in patients with ocular hypertension.?

The purpose of this study was to investigate the changes of calibre in retinal vessels in relation to body posture. Alterations in vessel calibre can be taken as an indicator of autoregulation occurring in the retinal vascular bed if other metabolic influences can be assumed to be constant. 


\section{Materials and methods}

Ten normal subjects (eight male and two female) aged 28-34 years were investigated. Subjects were excluded from the study if they suffered from any cardiovascular or respiratory disease or were taking any medication with cardiovascular effects. (One subject did suffer from migraine but had not had a recent attack and did not give rise to results which stood apart from the rest of the group.) All subjects were non-smokers. At the start of each study the subject was seated at the Zeiss (Oberkochen) fundus camera. All measurements were taken with the subject's pupil dilated (both for calculation of perfusion pressures and for vessel measurement).

After a period of cardiovascular stabilisation during which brachial blood pressure (mercury sphygmomanometer) and pulse rate were measured a series of five red-free fundus photographs centred on the optic disc were recorded. Each photograph was timed to mid-diastole by linking the camera shutter to the pulse recorded at the finger. At the end of each series of photographs the intraocular pressure was recorded with the Perkins hand-held tonometer. This method of tonometry was chosen to allow measurement of intraocular pressure in various body postures and in close temporal relationship to fundus photography. The subject was then asked to stand, and after a further period of cardiovascular stabilisation a further set of photographs was recorded and then the intraocular pressure. This procedure was then repeated with the subject lying on his/her right-hand side with the sphygmomanometer cuff on the upper arm. In all subjects the left eye was studied. Individuals with a refractive error greater than 3 dioptres were included in the study as the consequent difference in fundus magnification would not affect the proportional changes in calibre.

On a subsequent occasion the diastolic ophthalmic artery pressure was estimated. It was not possible to obtain this measurement at the same time as the photography was done, since the inevitable corneal distortion and drying associated with the application of the scleral suction cup was found to reduce the quality of the photographs. A modified Langham scleral suction cup was applied to the temporal sclera of the left eye with the subject seated at the fundus camera, thus reproducing the conditions of the photographic session and facilitating visualisation of retinal vessels. The suction pressure was increased until diastolic collapse of the arterial vessels at the disc was noted. Recordings were made three times in each of the three postures. The subject was then seated at the slit-lamp and the suction cup reapplied. The suction pressure was increased to the mean of the three suction pressures recorded at the camera for

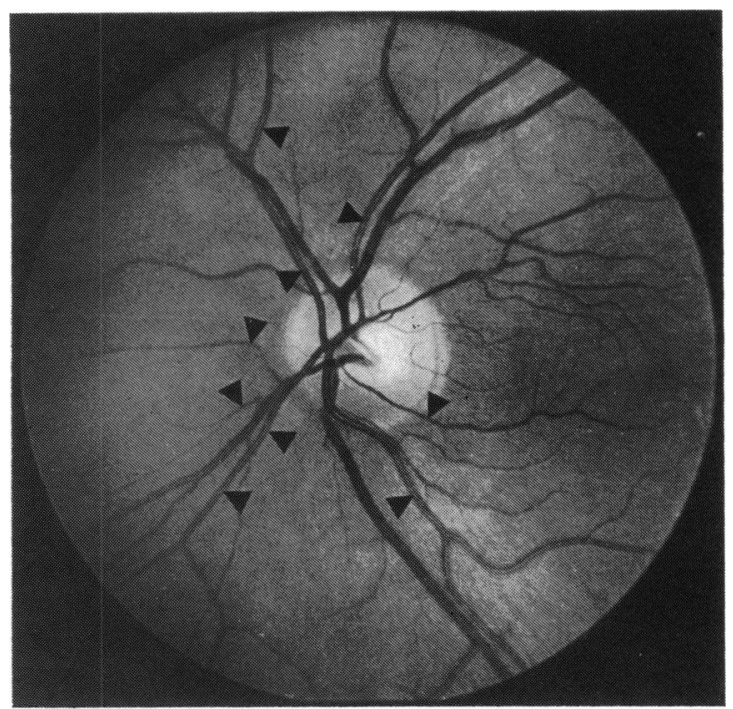

Fig. 1 A typical retinal photograph showing the sites of calibre measurements.

each posture and the corresponding intraocular pressure recorded with the Goldmann tonometer.

Measurements of retinal vessel calibre.were made with the Quantimet 800 Image Analyser (Cambridge Instruments) with $1 \mathrm{D}$ autoanalyser. Careful densitometric control of the photographs maintained the accuracy of the technique ${ }^{8}$ Calibre measurements were made at sites in the central portion of the photographic field, avoiding the optic disc (Fig. 1).

\section{Results}

POSTURE AND PERFUSION PRESSURES

Table 1 shows the ratio of the diastolic brachial blood pressure (DBP) and the intraocular pressure (Dyn P) corresponding to the diastolic dynamometry suction pressure for each posture in each subject. The DBP is the mean of the last four readings taken before photography in each posture. The mean ratios with their standard deviations (SD) are shown at the foot of Table 1 . There is a constant ratio in the sitting and standing postures $(0.62,0.65$ respectively), but the lying position produces a higher ratio (1.03) with a larger individual variation $(\mathrm{SD} \pm 0 \cdot 28)$.

Table 2 shows the estimated retinal artery perfusion pressures (PP). These perfusion pressures were the product of the mean Dyn P/DBP ratio (using the DBP recorded at the dynamometry session) and the diastolic blood pressure recorded during the photographic session, minus the intraocular pressure (also recorded during the photographic session). 
Table 1 Brachial diastolic and dynamometry pressures $(\mathrm{mmHg})$

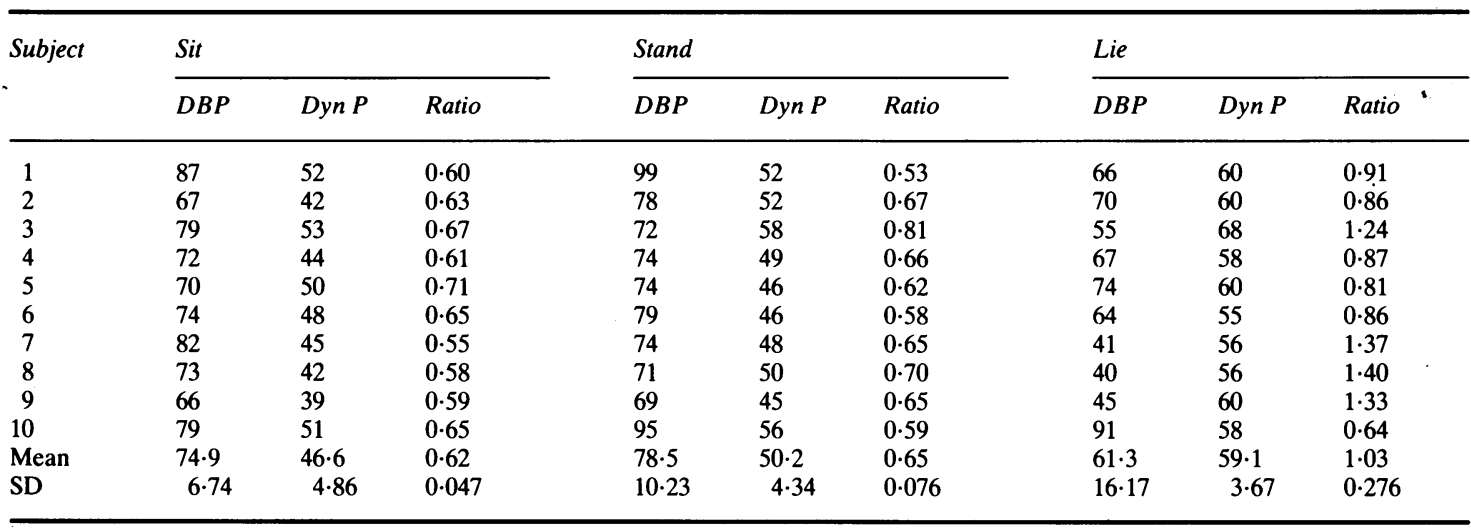

\section{ARTERIOLAR CALIBRE CHANGES}

A total of 71 arteriolar sites were measured with a range of 4-9 sites for each subject. Five replication photographs were measured for each posture in all but one instance, where only four replications were available. Measurements were recorded in $\mu \mathrm{m}$ at the image of the film. As regards the magnification factor for the Zeiss Fundus Camera, ${ }^{9} 1 \mathrm{~mm}$ on the film is equivalent to $0.45 \mathrm{~mm}$ on the fundus in the emmetropic eye; thus the mean calibre measured, 237.4 $\mu \mathrm{m}$ at the film, is equivalent to $106.8 \mu \mathrm{m}$ at the fundus.

A row and column analysis of variance (ANOV), with replications, was carried out for each subject, the rows representing different postures, the columns different sites. A significant $\mathrm{R} \times \mathrm{C}$ interaction was revealed in all but one subject (10), and in this case the variance within columns showed a highly significant difference $(p<0.001)$ by Bartlett's test. The results were therefore broken down by sites and tested by one-way ANOV, between and within

Table 2 Estimated perfusion pressures

\begin{tabular}{|c|c|c|c|c|c|}
\hline \multirow[t]{2}{*}{ Subject } & \multicolumn{3}{|c|}{ Perfusion pressure $(\mathrm{mmHg})$} & \multicolumn{2}{|c|}{$\%$ Change } \\
\hline & Sit & Stand & Lie & Sit Stand & Stand Lie \\
\hline 1 & 46 & 47 & 46 & $+2 \cdot 2$ & $-2 \cdot 1$ \\
\hline 2 & 32 & 43 & 58 & $+34 \cdot 4$ & $+34 \cdot 9$ \\
\hline 3 & 40 & 38 & 42 & $-5 \cdot 0$ & $+10 \cdot 5$ \\
\hline 4 & 33 & 38 & 52 & $+15 \cdot 2$ & $+36 \cdot 8$ \\
\hline 5 & 24 & 34 & 60 & $+41 \cdot 7$ & $+76 \cdot 5$ \\
\hline 6 & 39 & 42 & 51 & $+7 \cdot 7$ & $+21 \cdot 4$ \\
\hline 7 & 42 & 33 & 28 & $-21 \cdot 4$ & $-15 \cdot 2$ \\
\hline 8 & 31 & 33 & 25 & $+6 \cdot 5$ & $-24 \cdot 2$ \\
\hline 9 & 31 & 36 & 31 & $+16 \cdot 1$ & $-13 \cdot 9$ \\
\hline 10 & 31 & 49 & 77 & $+58 \cdot 1$ & $+57 \cdot 1$ \\
\hline \multicolumn{4}{|c|}{ Mean change } & $+15 \cdot 6$ & $+18 \cdot 2$ \\
\hline \multicolumn{4}{|c|}{$95 \%$ confidence limits } & $\pm 16 \cdot 8$ & \pm 23.8 \\
\hline
\end{tabular}

postures. Thirty one sites scattered among the 10 subjects showed significant variance between postures, tested by the $\mathrm{F}$ ratio at the $5 \%$ level. The confidence limits (CL) varied from site to site and are shown in a cumulative frequency histogram (Fig. 2). The left ordinate scale shows percentage change, referred to the mean of all calibres measured, the right ordinate scale the change in $\mu \mathrm{m}$ at the film; $50 \%$ of the significant changes were detected when the calibre altered by $6 \%$ or less, the median figure being $14.3 \mu \mathrm{m}$ at the film.

The least significant difference (LSD) for each one-way ANOV, was calculated at the 5\% level (LSD $=$ standard error, difference of means $\times t$, where $t$ has the degrees of freedom within the three postures), and those changes of calibre which exceeded this value were selected for further consideration. Derived from the 31 significant sites, a total of 18 changes in the 'sit-to-stand' posture shift, and 22 in the 'stand-to-lie' shift, were considered in the final analysis; their mean values and $95 \%$ confidence

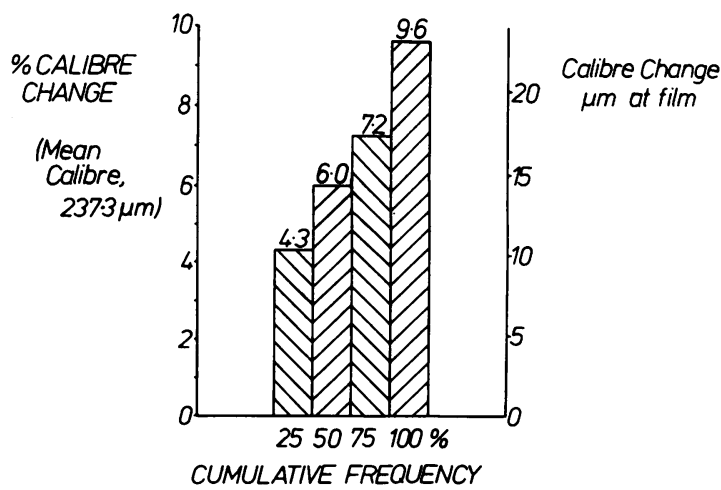

Fig. $25 \%$ confidence limits for significant arteriolar calibre changes. 


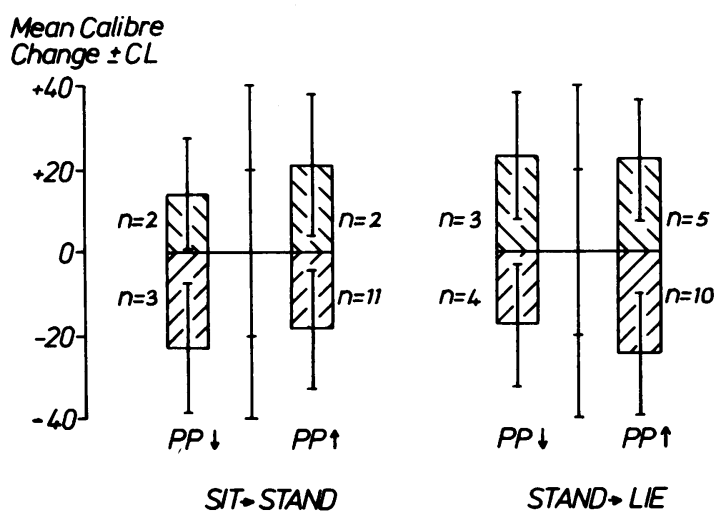

Fig. 3 Perfusion pressure and arteriolar calibre changes for the two posture changes studied.

limits are shown in Fig. 3. The results have been separated according to the accompanying perfusion pressure shift and the resultant calibre increase or decrease. The number of significant changes represented in each histogram bar is indicated, and the $95 \%$ confidence limits are shown by the line spanning the histogram block.

Because of the range of calibres of the arterioles measured (159-340 $\mu \mathrm{m}$ at the film), the proportional change is more relevant to possible physiological significance than is absolute calibre change, though the latter is appropriate for assessing statistical significance. Figs. 4 and 5 show the individual significant changes, expressed as percentages, plotted against the percentage perfusion pressure change for the posture alterations sit-to-stand and stand-to-lie respectively. Each plotted ordinate represents the changes in one subject and is shown with a different symbol. Only nine subjects are recorded in Fig. 4 as one (6) showed no significant changes on this posture shift.

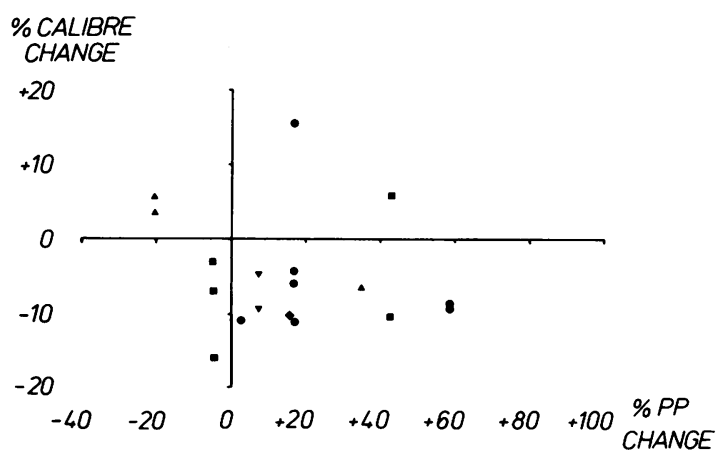

Fig. 4 Percentage perfusion pressure and arteriolar calibre change for the sit-to-stand posture change. Each column of symbols represents calibre changes for one subject.
An autoregulatory response would be represented by calibre decrease when the perfusion pressure rises and calibre increase when the perfusion pressure falls. Inspection of Fig. 4 shows 11 autoregulatory changes when the perfusion pressure rises and two when it falls; there are also five apparently nonautoregulatory changes where the calibre follows the increase or decrease in perfusion pressure. Similarly in Fig. 5 there are 10 autoregulatory changes with perfusion pressure increase and three associated with perfusion pressure decrease. There are also nine nonautoregulatory changes. The proportion of autoregulatory changes to non-autoregulatory changes when the perfusion pressure rises is smaller in the stand-to-lie shift $(10: 5)$ than in the sit-to-stand shift (11:2). In Fig. 5 three subjects show significant calibre changes in both directions, increase and decrease.

\section{Discussion}

Perfusion pressures have been recorded in terms of the diastolic blood pressure, as only the diastolic dynamometry pressure was recorded, to reduce the degree of disturbance to the background intraocular pressure produced by the scleral suction cup. The calibres also were measured in diastole to avoid complications of pulsatile variation.

The larger DBP/Dyn P ratio when lying (Table 1) can be explained in hydrostatic terms; for the vertical distance from heart to eyes is approximately $300 \mathrm{~mm}$ when sitting and standing, but abolished when lying, while the brachial sphygmomanometer cuff is about level with the heart when sitting and standing, but $100 \mathrm{~mm}$ above when lying to face the camera with the cuff on the upper arm. If these distances are scaled to equivalent pressure changes in $\mathrm{mmHg}$, the mean DBP lying must be increased $(61 \cdot 3+7 \mathrm{mmHg}$, and the mean Dyn P decreased (59.1-22 mmHg) to reach

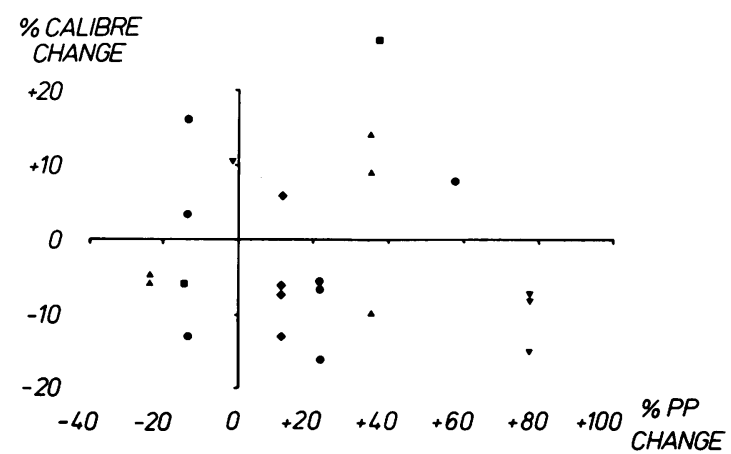

Fig. 5 Percentage perfusion pressure and arteriolar calibre change for the stand-to-lie posture change. Each column of symbols represents calibre changes for one subject. 
values equivalent to those in the sitting and standing positions. The resultant ratio after this adjustment, $0 \cdot 54$, is comparable to the original sitting and standing ratios.

Whatever technique is used to measure the apparent calibre of retinal vessels, the errors in measurement relate principally to the uncertainty of the vessel edge. They tend to be constant in absolute magnitude while increasing proportionally as the measured calibre decreases. For this reason statistical analysis was performed on the actual measurements of the film, though proportional, percentage changes were used at a later stage to aid comparison of significant vessels of different sizes.

A total of 142 calibre changes were recorded (two for each site). The mean value for an increase in calibre was $10.4 \mu \mathrm{m}$, SD 8.96 , and for decrease in calibre $12.7 \mu \mathrm{m}$, SD 9.61. Of the significant changes the median value for the LSD was $\pm 14.3 \mu \mathrm{m}$. This relatively large figure attributable to the collective errors of measurement from photography and image analysis accounts for the reduction from 71 sites measured to 31 sites showing significant changes at the $5 \%$ level.

While the majority of calibre changes ( 13 out of 18 , in the sit-to-stand shift, and 13 out of 22 in the standto-lie shift) accord with an autoregulatory response, calibre decreasing when the perfusion pressure rises, and increasing when it falls, there are a considerable number of non-autoregulatory responses, particularly following perfusion pressure rise in the stand-tolie shift which appear to be passive in nature, the calibre increasing with an increase of perfusion pressure. The presence of a considerable background variance makes it inevitable that a significant change of calibre can be detected only at those sites where a relatively large alteration has occurred. Nevertheless the confidence limits for the significant calibre changes (Fig. 3) fall well clear of the zero line in all but one instance, supporting the concept that distinct increases and decreases of calibre have occurred. Additional evidence for this conclusion is afforded by the row and column interaction in the two-way analysis of variance which reached the $0 \cdot 1 \%$ significance level in five subjects. This suggests that, even where individual calibre changes did not reach a significant level, there was a trend for changes in both directions to occur.
The topographical location of sites showing nonautoregulatory responses was examined and no repeatable pattern or particular regional distribution was found. Evidence for the mode of response of individual sites on successive changes in posture is fragmentary, but in subjects showing similar changes in perfusion pressure at each posture alteration three sites showed significant changes on both occasions, and in each instance the changes were dissimilar, the arteriole dilating on one occasion and constricting on the other. These observations suggest a random incidence of non-autoregulatory response, both in time and in topographical location.

Our investigation was originally planned to study the established state of the retinal circulation under altered conditions of perfusion pressure induced by changes in body posture: 26 out of 40 significant arteriolar calibre changes showed an autoregulatory response. The results of this study have not fully elucidated the nature of the arteriolar response, which will require further study.

Miss S Hague was supported by the Smith and Nephew Foundation. This study was supported by the Royal National Institute for the Blind. We are also grateful to colleagues at Moorfields Eye Hospital who participated in the study and to the technical staff of the Ophthalmology Department at the Royal College of Surgeons.

\section{References}

1 Harper AM. Physiology of cerebral blood flow. Br J Anaesth 1965; 37: 225-35.

2 Russell RWR. Evidence for autoregulation in the human retinal circulation. Lancet 1973 ; ii: 1048-50.

3 Dobree $\mathrm{JH}$. Calibre changes in retinal vessels occurring in raised ocular tension: circulatory compensation in chronic glaucoma. $\mathrm{Br}$ J Ophthalmol 1956; 40: 1-13.

4 Riva CE, Loebl M. Autoregulation of blood flow in the capillaries of the human macula. Invest Ophthalmol Vis Sci 1977; 16: 568-71.

5 Langham ME. Vascular pathophysiology of the ocular postural response - a pneumatometric study. Trans Ophthalmol Soc UK 1975; 95: 281-7.

6 Williams BI, Peart WS. Effect of posture on the intraocular pressure of patients with retinal vein obstruction. $\mathrm{BrJ}$ Ophthalmol 1978; 62: 688-93

7 Leonard TK, Kerr-Muir MG, Kirkby GR, Hitchings RA. Ocular hypertension and posture. BrJ Ophthalmol 1983; 67: 362-6.

8 Hill DW, Crabtree A. Vascular calibres. Trans Ophthalmol Soc UK 1984; 104: 107.

9 Norton HJ, Sullivan CT. On improving retinal photographic standards. Arch Ophthalmol 1958; 59: 746-51.

Accepted for publication 29 January 1987. 